\title{
Focused Interest Group on Microanalytical Standards (FIGMAS): An Update
}

\author{
Owen K. Neill ${ }^{1}$, Anette von der $\operatorname{Handt}^{2}$ and Julien M. Allaz ${ }^{3}$ \\ 1. Washington State University, Peter Hooper GeoAnalytical Laboratory, School of the Environment. \\ Pullman, WA, U.S.A. \\ 2. University of Minnesota Twin Cities, Department of Earth Sciences. Minneapolis, MN, U.S.A. \\ 3. University of Colorado Boulder, Department of Geological Sciences. Boulder, CO, U.S.A.
}

Reference materials and standards (RMS) play a crucial role in quantitative microbeam analysis, as they are the basis for instrumental calibration, data quality assurance (e.g., secondary standards) and interlaboratory comparison. Good standards that have been evaluated and certified for their homogeneity and reference composition are rare and only available from a handful of recognized providers. Whereas some crucial RMS have or will soon become unavailable, some researchers continue to develop and test new RMS [1-3], at times becoming more widely disseminated. Unfortunately, practical constraints make RMS development often difficult [4], and leave analysts with a set of "second choice" reference materials instead of certified standards. Such reference materials may suffer from various shortcomings such as questionable provenance, homogeneity or impurity issues (natural samples), contamination (synthetic materials), or incomplete or inaccurate reference compositions. Furthermore, variations between individual batches and/or updated reference compositions [1,5]) can lead to confusion and the potential risk for mixing old and new values. Efforts have to be made to keep a record, evaluate, and guarantee the quantity of RMS for the next century.

Intimate knowledge and vetting of one's own RMS collection, awareness of compositional variations between standard batches [6], and adhering to proper standard maintenance protocols [7] are key components in this efforts. This is specifically important as failure in any of these aspects can turn originally "good" RMS to what is considered "bad" or "ugly" [7]. Metals that would otherwise be homogeneous and pure can easily oxidize, while well-characterized glasses can be altered by electron beam damage. Some RMS may have subpopulations that deviate from the published composition, and one has to identify such outliers. To return a "bad" RMS to the "good" (or at least better) category, individual labs may need to adjust reference composition on file to accurately reflect true compositions. In other cases, a modification of the analytical protocol might be necessary, e.g., defocussing the electron beam to minimize the effect of sub-micrometer inclusions [8], averaging over multiple grains to minimize bias due to intergrain heterogeneity [9], using intensity corrections to account for beam damage effects [10], or accounting for peak shifts or shape changes between materials [11].

Generation and upkeep of this level of documentation for a wide range of materials is often beyond the scope of what a single lab or RMS provider can perform due to the dispersed nature of information, often scattered over individual publications, conference abstracts, and personal anecdotes. Furthermore, recent hardware innovations such as brighter electron sources (Schottky emitters), new WDS monochromators, modern SDD-EDS, and a new generation of soft X-ray spectrometers (SXES, iZPS) will necessitate further characterization of new and established reference materials. Software innovations are establishing new benchmarks in terms of improved precision, accuracy and efficiency.

To respond to the increasing need for consolidation of existing and emerging information about standards and reference materials, the Focused Interest Group on Microanalytical Standards (FIGMAS 
[12]: https://figmas.org) was founded in 2015 under the umbrella of both Microanalysis Society (MAS) and Microscopy Society of America (MSA). Key component of the FIGMAS's efforts is currently the creation of a web-based database that strives to improve access to RMS information and address the aforementioned issues. This database can provide a fresh avenue to disseminate information, establish guidelines for critical evaluation of one's own standards and help make informed decisions for future acquisitions of standard materials.

We will provide an update on the current state of this web-based database and the information it collects on published and unpublished RMS. This database is continuously growing and the entries are peerreviewed by FIGMAS members.

\section{References:}

[1] JJ Donovan et al., Can. Mineral, 41 (2003), p. 221-232

[2] PK Carpenter P K et al., J. Res. Nat. Inst. Stand. Tech. 107 (2002), p. 703-718

[3] EP Vicenzi, J. Res. Nat. Inst. Stand. Tech. 107 (2002), p. 719-727

[4] RB Marinenko, J. Res. Nat. Inst. Stand. Tech. 107 (2002), p. 687-691

[5] B Wagner B, Anal. Bioanal. Chem. 402 (2012), p. 1667-1677

[6] J Fournelle, AGU Fall Meeting Abstracts V31E-2009 (2009).

[7] PK Carpenter, Microsc. Microanal. 14 (2008), p. 530-531.

[8] PK Carpenter and EP Vicenzi, Microsc. Microanal. 18 (2012), p. 1734-1735.

[9] J Fournelle, AGU Fall Meeting Abstracts V23C-2827 (2012).

[10] JJ Donovan, Topical Conf. Microanalytical Reference Materials (2012).

[11] J Fournelle and CA Geiger, AGU Fall Meeting abstract V51C-2208 (2010).

[12] JM Allaz, et al., Microsc. Microanal. 21 (2015), p. 2019-2020.

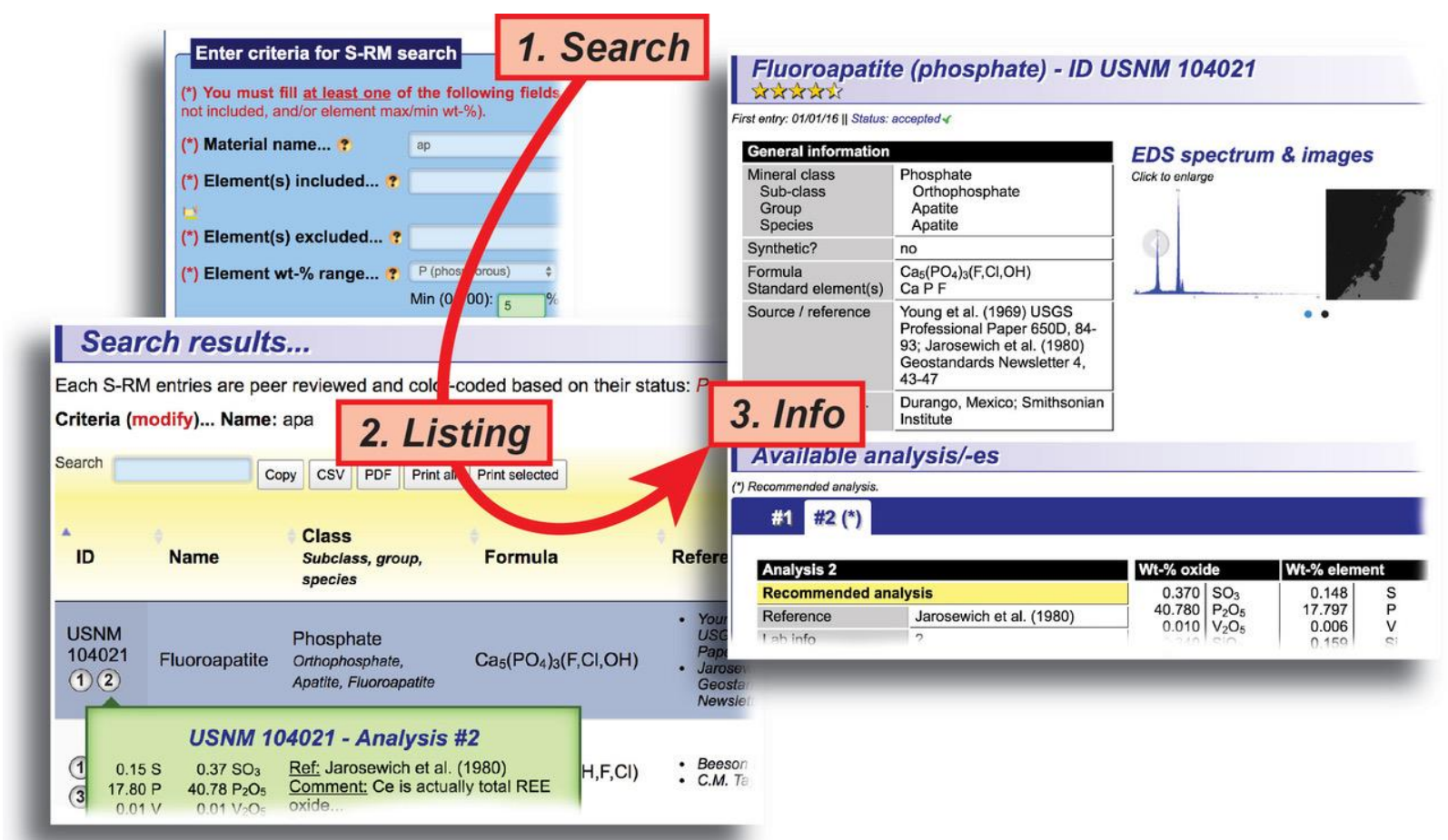

Figure 1: Screen shot of FIGMAS database currently in development (more details at https://figmas.org/std/std_info.php) 